\title{
Front Matter: Volume 8667
}

, "Front Matter: Volume 8667," Proc. SPIE 8667, Multimedia Content and Mobile Devices, $86671 Z$ (4 April 2013); doi: 10.1117/12.2021286

SPIE Event: IS\&T/SPIE Electronic Imaging, 2013, Burlingame, California, United SPIE. States 


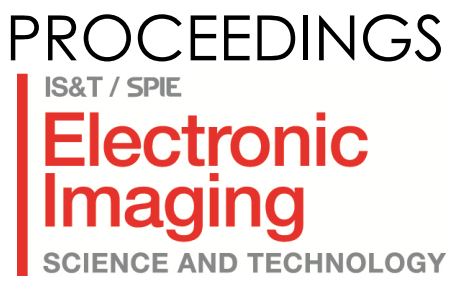

\title{
Multimedia Content and Mobile Devices
}

\author{
David Akopian \\ Reiner Creutzburg \\ Todor G. Georgiev \\ Lyndon S. Kennedy \\ Andrew Lumsdaine \\ Kevin J. Matherson \\ Nicu Sebe \\ Cees G. M. Snoek \\ Dietmar Wüller \\ Editors
}

4-6 February 2013

Burlingame, California, United States

Sponsored by

IS\&T-The Society for Imaging Science and Technology

SPIE

Cosponsored by

Qualcomm Inc. (United States)

Published by

SPIE 
The papers included in this volume were part of the technical conference cited on the cover and title page. Papers were selected and subject to review by the editors and conference program committee. Some conference presentations may not be available for publication. The papers published in these proceedings reflect the work and thoughts of the authors and are published herein as submitted. The publishers are not responsible for the validity of the information or for any outcomes resulting from reliance thereon.

Please use the following format to cite material from this book:

Author(s), "Title of Paper," in Multimedia Content and Mobile Devices, edited by David Akopian, Reiner Creutzburg, Todor G. Georgiev, Lyndon S. Kennedy, Andrew Lumsdaine, Kevin J. Matherson, Nicu Sebe, Cees G. M. Snoek, Dietmar Wüller, Proceedings of SPIE-IS\&T Electronic Imaging, SPIE Vol. 8667. Article CID Number (2013)

ISSN: 0277-786X

ISBN: 9780819494405

Copublished by

SPIE

P.O. Box 10, Bellingham, Washington $98227-0010$ USA

Telephone +1 3606763290 (Pacific Time) · Fax +1 3606471445

SPIE.org

and

IS\&T-The Society for Imaging Science and Technology

7003 Kilworth Lane, Springfield, Virginia, 22151 USA

Telephone +1 7036429090 (Eastern Time) · Fax +1 7036429094

imaging.org

Copyright (C) 2013, Society of Photo-Optical Instrumentation Engineers and The Society for Imaging Science and Technology.

Copying of material in this book for internal or personal use, or for the internal or personal use of specific clients, beyond the fair use provisions granted by the U.S. Copyright Law is authorized by the publishers subject to payment of copying fees. The Transactional Reporting Service base fee for this volume is $\$ 18.00$ per article (or portion thereof), which should be paid directly to the Copyright Clearance Center (CCC), 222 Rosewood Drive, Danvers, MA 01923. Payment may also be made electronically through CCC Online at copyright.com. Other copying for republication, resale, advertising or promotion, or any form of systematic or multiple reproduction of any material in this book is prohibited except with permission in writing from the publisher. The CCC fee code is 0277-786X/13/\$18.00.

Printed in the United States of America.

Paper Numbering: Proceedings of SPIE follow an e-First publication model, with papers published first online and then in print and on CD-ROM. Papers are published as they are submitted and meet publication criteria. A unique, consistent, permanent citation identifier (CID) number is assigned to each article at the time of the first publication. Utilization of CIDs allows articles to be fully citable as soon as they are published online, and connects the same identifier to all online, print, and electronic versions of the publication. SPIE uses a six-digit CID article numbering system in which:

- The first four digits correspond to the SPIE volume number.

- The last two digits indicate publication order within the volume using a Base 36 numbering

system employing both numerals and letters. These two-number sets start with 00, 01, 02, 03, 04 , $05,06,07,08,09,0 \mathrm{~A}, 0 \mathrm{~B} \ldots \mathrm{.0Z}$, followed by 10-1Z, 20-2Z, etc.

The CID Number appears on each page of the manuscript. The complete citation is used on the first page, and an abbreviated version on subsequent pages. Numbers in the index correspond to the last two digits of the six-digit CID Number. 


\section{Contents}

ix Conference Committee

JOURNAL OF ELECTRONIC IMAGING FOCAL TRACK PRESENTATIONS

866701 Introduction to the JEl Focal Track Presentations

T. Georgiev, Qualcomm Inc. (United States); A. Lumsdaine, Indiana Univ. (United States)

Resolution and sensitivity of wafer-level multi-aperture cameras [8667-66]

A. Oberdörster, Fraunhofer Institute for Applied Optics and Precision Engineering

(Germany); H. P. A. Lensch, Eberhard Karls Univ. Tübingen (Germany)

(J. Electron. Imaging. 22, 0011001 )

Refinement of depth maps by fusion of multiple estimates [8667-67]

B. Krishnamurthy, A. Rastogi, Adobe Systems India Pvt. Ltd. (India)

(J. Electron. Imaging. 22, 0011002)

Image deblurring in smarphone devices using built-in intertial measurement sensors [8667-52]

O. Šindelár̆, Charles Univ. in Prague (Czech Republic); F. Šroubek, Institute of Information

Theory and Automation AS CR, v.v.i. (Czech Republic) (J. Electron. Imaging. 22, 001 1003)

Compensating specular highlights for non-Lambertian projection surfaces [8667-53]

C.-T. Kao, T.-H. Huang, National Taiwan Univ. (Taiwan); H. Lee, Univ. of California, Santa Barbara (United States); H. H. Chen, National Taiwan Univ. (Taiwan)

(J. Electron. Imaging. 22, 0011004)

\section{PART A Multimedia Content Access: Algorithms and Systems VII}

\section{SPECIAL SESSION ON MULTIMEDIA EVENT DETECTION}

866706 Sparse conditional mixture model: late fusion with missing scores for multimedia event detection [8667-4]

R. M. Nallapati, E. Yeh, G. Myers, SRI International (United States)

866707 Can object detectors aid internet video event retrieval? [8667-5]

D. Modolo, C. G. M. Snoek, Univ. of Amsterdam (Netherlands)

866708 Multimedia event detection using visual concept signatures [8667-6]

E. Younessian, M. Quinn, T. Mitamura, A. Hauptmann, Carnegie Mellon Univ. (United States) 
866709 A fast approach for integrating ORB descriptors in the bag of words model [8667-7]

C. Grana, D. Borghesani, M. Manfredi, R. Cucchiara, Univ. degli Studi di Modena e Reggio Emilia (Italy)

8667 OA Video-based analysis of motion skills in simulation-based surgical training [8667-8]

Q. Zhang, L. Chen, Q. Tian, B. Li, Arizona State Univ. (United States)

8667 OC Exploiting visual search theory to infer social interactions [8667-10]

P. Rota, D.-T. Dang-Nguyen, N. Conci, N. Sebe, Univ. of Trento (Italy)

\section{BAY AREA MULTIMEDIA AND BEYOND}

8667 OE Presentation video retrieval using automatically recovered slide and spoken text [8667-12] M. Cooper, FX Palo Alto Lab. (United States)

8667 OF VidCat: an image and video analysis service for personal media management [8667-13] L. Begeja, E. Zavesky, Z. Liu, D. Gibbon, R. Gopalan, B. Shahraray, AT\&T Labs. Research (United States)

INTERACTIVE PAPER SESSION

8667 OG Audio stream classification for multimedia database search [8667-14]

M. Artese, ITC-Consiglio Nazionale delle Ricerche (Italy); S. Bianco, Univ. di Milano-Bicocca (Italy); I. Gagliardi, ITC-Consiglio Nazionale delle Ricerche (Italy); F. Gasparini, Univ. di Milano-Bicocca (Italy)

$8667 \mathrm{OH}$ Structuring a sharded image retrieval database [8667-15]

E. Liang, A. Zakhor, Univ. of California, Berkeley (United States)

86670 Diversification of visual media retrieval results using saliency detection [8667-16]

O. Muratov, G. Boato, F. B. De Natale, Univ. of Trento (Italy)

\section{PART B Multimedia on Mobile Devices 2013}

\section{SESSION 4}

8667 0J Enabling customer self service through image processing on mobile devices [8667-17] I. Kliche, Telekom Innovation Labs. (Germany); S. Hellmann, Brandenburg Univ. of Applied Sciences (Germany); J. Kreutel, Beuth Univ. of Applied Sciences (Germany)

8667 OK Cognitive styles and visual quality [8667-18]

S. Jumisko-Pyykkö, Tampere Univ. of Technology (Finland); D. Strohmeier, Technische Univ. Berlin (Germany) 
8667 OL Subjective evaluation of HEVC in mobile devices [8667-19]

R. Garcia, H. Kalva, Florida Atlantic Univ. (United States)

SESSION 5

8667 OM Location-based tracking using long-range passive RFID and ultrawideband communications (Invited Paper) [8667-20]

F. Nekoogar, F. Dowla, Lawrence Livermore National Lab. (United States)

8667 ON Real-time content-aware video retargeting for tunnel vision assistance [8667-21]

T. Knack, A. Savakis, Rochester Institute of Technology (United States)

866700 Human movement activity classification approaches that use wearable sensors and mobile devices [8667-22]

S. Kaghyan, Armenian-Russian (Slavonic) Univ. (Armenia); H. Sarukhanyan, Institute for Informatics and Automation Problems (Armenia); D. Akopian, The Univ. of Texas at San Antonio (United States)

$8667 \mathrm{OP}$ Concept for practical exercises for studying autonomous flying robots in a university environment: Part I [8667-23]

R. Band, J.-S. Pleban, S. Schön, R. Creutzburg, A. Fischer, Brandenburg Univ. of Applied Sciences (Germany)

$86670 Q$ Applications of multimedia technology on autonomous flying robots for university technology transfer projects [8667-24]

S. Schön, R. Band, J.-S. Pleban, R. Creutzburg, A. Fischer, Brandenburg Univ. of Applied Sciences (Germany)

\section{SESSION 6}

8667 OS Digitized forensics: retaining a link between physical and digital crime scene traces using QR-codes [8667-26]

M. Hildebrandt, S. Kiltz, J. Dittmann, Otto-von-Guericke-Univ. Magdeburg (Germany)

8667 OT Smart apps for applied machine learning on mobile devices: the MOMO project [8667-27]

S. Edlich, M. Vogler, Beuth Univ. of Techology Berlin (Germany)

8667 OU Real-time volume rendering of digital medical images on an iOS device [8667-28]

C. Noon, BodyViz.com (United States); J. Holub, E. Winer, lowa State Univ. (United States)

8667 OV MessageSpace: a messaging system for health research [8667-29]

R. D. Escobar, D. Akopian, The Univ. of Texas at San Antonio (United States);

D. Parra-Medina, L. Esparza, The Univ. of Texas Health Science Ctr. at San Antonio (United States)

8667 OW Multi-resolution edge detection with edge pattern analysis [8667-30]

B. Jiang, National Institute of Aerospace (United States) 
8667 0X Client-side Skype forensics: an overview [8667-31]

T. Meißner, K. Kröger, R. Creutzburg, Brandenburg Univ. of Applied Sciences (Germany)

$86670 Z$ Gradient-based fusion of infrared and visual face images using support vector machine for human face identification [8667-33]

P. Saha, M. K. Bhowmik, Tripura Univ. (India); D. Bhattacharjee, Jadavpur Univ. (India);

B. K. De, Tripura Univ. (India); M. Nasipuri, Jadavpur Univ. (India)

866710 Future mobile access for open-data platforms and the BBC-DaaS system [8667-34]

S. Edlich, S. Singh, I. Pfennigstorf, Beuth Univ. of Technology Berlin (Germany)

866712 Location tracking forensics on mobile devices [8667-36]

S. Sack, K. Kröger, R. Creutzburg, Brandenburg Univ. of Applied Sciences (Germany)

866713 Conception of a course for professional training and education in the field of computer and mobile forensics: Part II: Android Forensics [8667-37]

K. Kröger, R. Creutzburg, Brandenburg Univ. of Applied Sciences (Germany)

866714 Possibilities and modification of the forensic investigation process of solid-state drives [8667-38]

F. Irmler, K. Kröger, R. Creutzburg, Brandenburg Univ. of Applied Sciences (Germany)

866715 Mobile learning in medicine [8667-40]

S. Serkan Güllüoğlu, Istanbul Arel Univ. (Turkey)

866716 Overview and forensic investigation approaches of the gaming console Sony PlayStation Portable [8667-41]

S. Schön, R. Schön, K. Kröger, R. Creutzburg, Brandenburg Univ. of Applied Sciences (Germany)

866718 Reconstruction of the image on the Cartesian lattice from a finite number of projections in computed-tomographic imaging [8667-74]

N. Du, Y. Feng, A. M. Grigoryan, The Univ. of Texas at San Antonio (United States)

866719 Method of G-particles for image reconstruction from a finite number of projections [8667-75]

A. M. Grigoryan, N. Du, The Univ. of Texas at San Antonio (United States)

\section{PART C Mobile Imaging System Design and Image Quality}

\section{OBJECT CLASSIFICATION AND REMOTE SENSING I}

8667 1B Determination of sensor oversize for stereo-pair mismatch compensation and image stabilization [8667-45]

P. Kulkarni, Silicon Image Inc. (United States) 
8667 1C Nokia PureView oversampling technology (Invited Paper) [8667-77]

T. Vuori, J. Alakarhu, E. Salmelin, A. Partinen, Nokia Corp. (Finland)

8667 1D Image quality evaluation using moving targets [8667-46]

U. Artmann, Image Engineering GmbH \& Co. KG (Germany)

$8667 \mathrm{lE}$ Multiple-field approach for aberration correction in miniature imaging systems based on wafer-level production [8667-47]

E. Logean, T. Scharf, N. Bongard, H. P. Herzig, Ecole Polytechnique Fédérale de Lausanne

(Switzerland); M. Rossi, Heptagon (Switzerland)

8667 1G Auto-focus algorithm based on statistical blur estimation [8667-49]

P. Kulkarni, Silicon Image Inc. (United States)

IMAGE QUALITY EVALUATION METHODS/STANDARDS FOR MOBILE AND DIGITAL

PHOTOGRAPHY I: JOINT SESSION WITH CONFERENCES 8653, 8660, AND 8667C

$86671 \mathrm{H} \quad$ Low light performance of digital still cameras [8667-50]

D. Wueller, Image Engineering GmbH \& Co. KG (Germany)

IMAGE QUALITY EVALUATION METHODS/STANDARDS FOR MOBILE AND DIGITAL PHOTOGRAPHY II: JOINT SESSION WITH CONFERENCES 8653, 8660, AND 8667C

866711 Noise evaluation standard of image sensor using visual spatio-temporal frequency characteristics [8667-51]

T. Fujii, S. Suzuki, S. Saito, Sony Corp. (Japan)

\section{PART D Mobile Computational Photography}

\section{KEYNOTE SESSION}

$86671 \mathrm{~J}$ Lytro camera technology: theory, algorithms, performance analysis (Keynote Paper) [8667-70]

T. Georgiev, Qualcomm Inc. (United States); Z. Yu, Univ. of Delaware (United States);

A. Lumsdaine, Indiana Univ. (United States); S. Goma, Qualcomm Inc. (United States)

PLENOPTIC CAMERAS: THEORY

$8667 \mathrm{lL}$ Wave analysis of a plenoptic system and its applications [8667-55]

S. A. Shroff, K. Berkner, Ricoh Innovations Inc. (United States)

$86671 \mathrm{M}$ Fourier analysis of the focused plenoptic camera [8667-56]

A. Lumsdaine, L. Lin, J. Willcock, Y. Zhou, Indiana Univ. (United States) 
IMAGE PROCESSING

8667 iN Design of user interfaces for selective editing of digital photos on touchscreen devices [8667-57]

T. Binder, M. Steiding, M. Wille, N. Kokemohr, Google (Germany)

866710 Touch HDR: photograph enhancement by user controlled wide dynamic range adaptation [8667-58]

S. Verrall, H. Siddiqui, K. Atanassov, S. Goma, V. Ramachandra, Qualcomm Inc. (United States)

8667 IP Temporal image stacking for noise reduction and dynamic range improvement [8667-59] K. Atanassov, J. Nash, S. Goma, V. Ramachandra, H. Siddiqui, Qualcomm Inc. (United States)

$86671 Q \quad$ Accelerating defocus blur magnification [8667-60]

F. Kriener, T. Binder, M. Wille, Google (Germany)

PLENOPTIC CAMERAS: DEPTH OF FIELD

8667 IR Adaptive DOF for plenoptic cameras [8667-62]

A. Oberdörster, Fraunhofer Institute for Applied Optics and Precision Engineering (Germany); H. P. A. Lensch, Eberhard Karls Univ. Tübingen (Germany)

8667 is Plenoptic depth map in the case of occlusions [8667-63]

Z. YU, J. YU, Univ. of Delaware (United States); A. Lumsdaine, Indiana Univ. (United States);

T. Georgiev, Qualcomm Inc. (United States)

8667 IT Reduced depth of field using multi-image fusion [8667-64]

B. Ajdin, T. Ahonen, Nokia Research Ctr. (United States)

$86671 \mathrm{U}$ Optimizing depth-of-field based on a range map and a wavelet transform [8667-65] M. Wellner, Pattern Recognition Co. GmbH (Germany); T. Käster, Pattern Recognition Co. GmbH (Germany) and Univ. of Lübeck (Germany); T. Martinetz, E. Barth, Univ. of Lübeck (Germany)

IMAGE TRACKING AND STABILIZATION

8667 IW A new fusion-based low light still-shot stabilization [8667-71]

Y.-S. Moon, S.-H. Lee, SAMSUNG Electronics Co., Ltd. (Korea, Republic of)

8667 1X Real-time skeleton tracking for embedded systems [8667-72]

F. Coleca, Univ. of Lübeck (Germany) and Gestigon GmbH (Germany); S. Klement,

Gestigon GmbH (Germany); T. Martinetz, E. Barth, Univ. of Lübeck (Germany)

Author Index

viii 


\title{
Conference Committee
}

\author{
Symposium Chair
}

Gaurav Sharma, University of Rochester (United States)

Symposium Cochair

Sergio R. Goma, Qualcomm Inc. (United States)

\section{Part A Multimedia Content Access: Algorithms and Systems VII}

Conference Chairs

Cees G. M. Snoek, Universiteit van Amsterdam (Netherlands)

Nicu Sebe, Universitá degli Studi di Trento (Italy)

Lyndon S. Kennedy, Yahoo! Inc. (United States)

\section{Conference Program Committee}

John Adcock, FX Palo Alto Laboratory (United States)

Noboru Babaguchi, Osaka University (Japan)

Tat-Seng Chua, National University of Singapore (Singapore)

Matthew L. Cooper, FX Palo Alto Laboratory (United States)

Francesco G. B. De Natale, Universitá degli Studi di Trento (Italy)

Alberto Del Bimbo, Universitá degli Studi di Firenze (Italy)

Jianping Fan, The University of North Carolina at Charlotte (United States)

Yuli Gao, Google (United States)

Alan Hanjalic, Technische Universiteit Delft (Netherlands)

Alexander G. Hauptmann, Carnegie Mellon University (United States)

Winston H. Hsu, National Taiwan University (Taiwan)

Gang Hua, Stevens Institute of Technology (United States)

Xian-Sheng Hua, Microsoft Research Asia (China)

Yu-Gang Jiang, Fudan University (China)

Paul H. Lewis, University of Southampton (United Kingdom)

Xirong Li, Universiteit van Amsterdam (Netherlands)

Rainer W. Lienhart, Universität Augsburg (Germany)

Qian Lin, Hewlett-Packard Laboratories (United States)

Vasileios Mezaris, Informatics and Telematics Institute (Greece)

Chong-Wah Ngo, City University of Hong Kong (Hong Kong, China)

Yong Rui, Microsoft Corporation (China)

Alan F. Smeaton, Dublin City University (Ireland) 
John R. Smith, IBM Thomas J. Watson Research Center (United States) Hari Sundaram, Arizona State University (United States)

Qi Tian, The University of Texas at San Antonio (United States)

Dong Wang, Hulu (United States)

Meng Wang, National University of Singapore (Singapore)

Changsheng $\mathrm{Xu}$, Institute of Automation (China)

Rong Yan, Facebook Inc. (United States)

Jun Yang, Facebook Inc. (United States)

\section{Session Chairs}

Special Session on Multimedia Event Detection

Cees G. M. Snoek, Universiteit van Amsterdam (Netherlands)

Semantic Multimedia Content Analysis

Lyndon S. Kennedy, Yahoo! Inc. (United States)

Bay Area Multimedia and Beyond

Lyndon S. Kennedy, Yahoo! Inc. (United States)

\section{Part B Multimedia on Mobile Devices 2013}

\section{Conference Chairs}

Reiner Creutzburg, Fachhochschule Brandenburg (Germany)

David Akopian, The University of Texas at San Antonio (United States)

\section{Conference Program Committee}

Sos S. Agaian, The University of Texas at San Antonio (United States)

Faouzi Alaya Cheikh, Gjøvik Universitetet College (Norway)

Nina T. Bhatti, Hewlett-Packard Laboratories (United States)

Linda Breitlauch, Mediadesign Hochschule für Design und Informatik (Germany)

Chang Wen Chen, University at Buffalo (United States)

Philip C. L. Chen, University of Macau (Macao, China)

David Cook, The British Institute of Technology \& E-commerce (United Kingdom)

Kenneth J. Crisler, Motorola, Inc. (United States)

Stefan Edlich, Technische Fachhochschule Berlin (Germany)

Atanas P. Gotchev, Tampere University of Technology (Finland)

Lajos Hanzo, University of Southampton (United Kingdom)

Zhihai He, University of Missouri-Columbia (United States)

Louis J. Kerofsky, Sharp Labs. of America, Inc. (United States)

Hendrik O. Knoche, University College London (United Kingdom) 
Ramanujan Venkata Krishnan, The University of Texas Health Science Center at San Antonio (United States)

Catalin Lacatus, Qualcomm, Inc. (United States)

Xin Li, West Virginia University (United States)

Gabriel G. Marcu, Apple Inc. (United States)

Manzur M. Murshed, Monash University (Australia)

Sethuraman A. Panchanathan, Arizona State University (United States)

Kari A. Pulli, NVIDIA Corporation (United States)

Matthias Rauterberg, Technische Universiteit Eindhoven (Netherlands)

Phillip A. Regalia, TELECOM \& Management SudParis (France)

René Rosenbaum, University of California, Davis (United States)

Phanikrishna K. Sagiraju, The University of Texas at San Antonio (United States)

Abhay Samant, NI Systems (India) Pvt. Ltd. (India)

Olli Silvén, University of Oulu (Finland)

Jarmo Henrik Takala, Tampere University of Technology (Finland)

Marius Tico, Apple, Inc. (United States)

Haitao Zheng, University of California, Santa Barbara (United States)

\section{Part C Mobile Imaging System Design and Image Quality}

\section{Conference Chairs}

Dietmar Wüller, Image Engineering GmbH \& Company KG (Germany)

Kevin J. Matherson, Hewlett-Packard Company (United States)

Conference Program Committee

Donald J. Baxter, STMicroelectronics Ltd. (United Kingdom)

Sergio R. Goma, Qualcomm Inc. (United States)

Paul M. Hubel, Apple Inc. (United States)

George John, Microsoft Corporation (United States)

Jon S. McElvain, Dolby Laboratories, Inc. (United States)

Ricardo J. Motta, NVIDIA Corporation (United States)

Joni Oja, Nokia Research Center (Finland)

\section{Session Chairs}

Object Classification and Remote Sensing I

Kevin J. Matherson, Hewlett-Packard Company (United States)

Object Classification and Remote Sensing II

Paul M. Hubel, Apple Inc. (United States) 
Image Quality Evaluation Methods/Standards for Mobile and Digital Photography I: Joint Session with Conferences 8653, 8660, and 8667C Sebastiano Battiato, Universitá degli Studi di Catania (Italy)

Peter D. Burns, Burns Digital Imaging (United States)

Image Quality Evaluation Methods/Standards for Mobile and Digital Photography II: Joint Session with Conferences 8653, 8660, and 8667C Dietmar Wüller, Image Engineering GmbH \& Co. KG (Germany)

Nitin Sampat, Rochester Institute of Technology (United States)

\section{Part D Mobile Computational Photography}

\section{Conference Chairs}

Todor G. Georgiev, Qualcomm Inc. (United States)

Andrew Lumsdaine, Indiana University (United States)

\section{Conference Program Committee}

Raja Bala, Xerox Corporation (United States)

Erhardt Barth, Universität zu Lübeck (Germany)

Kathrin Berkner, Ricoh Innovations, Inc. (United States)

Charles A. Bouman, Purdue University (United States)

David J. Brady, Duke University (United States)

Paolo Favaro, Heriot-Watt University (United Kingdom)

Sergio R. Goma, Qualcomm Inc. (United States)

Sylvain Paris, Adobe Systems Inc. (United States)

Shmuel Peleg, The Hebrew University of Jerusalem (Israel)

Kari A. Pulli, NVIDIA Corporation (United States)

Amnon Shashua, The Hebrew University of Jerusalem (Israel)

Sabine Süsstrunk, Ecole Polytechnique Fédérale de Lausanne (Switzerland)

Ashok Veeraraghavan, Rice University (United States)

Edwin Wolterink, Anteryon B.V. (Netherlands)

Jingyi Yu, University of Delaware (United States) 